\title{
IR THERMOGRAPHY IN INTERVENTIVE CARDIOLOGY PRACTICE
}

\author{
V.Veikutis, T. Mickevicius, K. Morkunaite ${ }^{\star \star}$, M. Viezelis***, D. Vaitkaitis*, P. Dobozinskas*
}

Lithuanian University of Health Sciences, Institute of Cardiology, vincentas.veikutis@med.kmu.lt

* Lithuanian University of Health Sciences, Dept of Disaster Medicine;

** Lithuanian University of Health Sciences, Kaunas, Institute of Cardiology; Clinic of Cardiology;

*** Lithuanian University of Health Sciences, Kaunas, Lithuania

\section{Introduction}

In over then 20 years of clinical experience, catheter radiofrequency ablation (RFA) has been in standard used to correct for many kinds of cardiac arrhythmias. Unfortunately, despite of increased experience and using modern RFA technologies short- and long-range postoperative complications and relapses fluctuate from 0,5 to 14 percent. Consequently, efficacy must be balance against the safety and possibly potential complications. Surgical trauma or RFA in heart zones with high level of vagosympathetic innervation often determine various grade atrioventricular blocks, untreatable or difficultly corrected supraventricular and ventricular tachycardias. However, reasons of these post ablative complications are not analysed and usually comments contains only technical parameters of navigation equipment and ablative electrodes. Aim of our study was to analyse possible mechanisms of these complications and determine optimal RFA parameters, performing endocardial destructive procedures in various structures of heart.

\section{Methods}

10 female pigs (weight $\sim 20-25 \mathrm{~kg}$ ) used in experimental study. Heart exposed via sternotomy and pericardtomy, destruction of various heart areas was performed from the endocardial surface by inserting the electrode through the incision on the right or left auricle. Standard 4 and $8 \mathrm{~mm}$ "Biosense Webster"(Johnson\&Johnson,USA) intracardial electrodes were used. Destruction was performed using Prucka Engineering® RF energy generator OSYPKA HAT 200S. Destruction parameters: energy - 10-50 W, time - 10-60 sec. Temperature changes in epicardial surface registered with thermo camera ThermaCAM $P 640$, emission $\dot{\varepsilon}-0.98$, temperature sensitivity $\leq 0.05^{\circ} \mathrm{C}$. After destruction, damaged zones were excised and evaluated histological. Three excised hearts were given for anatomorphological analysis - intracardial nerve tracts topography and state identification.

\section{Results}

After start of RFA procedure on atrium tissue destructive effect $\left(>46^{\circ} \mathrm{C}\right)$ was observed in $5-7 \mathrm{~s}$ period, independently from power of energy and type of electrode. Dotted zone of total destruction using $20-30 \mathrm{~W}$ power and both type electrodes was $3-4 \mathrm{~mm}$, whereas using $50 \mathrm{~W}-4-7 \mathrm{~mm}$. Partial destruction zone usually was equal or marginally, but regularly major than total destruction zone. Usually zone of destruction was ellipsoid, whereas temperature restoration occurred in $25-78$ s., subject to power of energy, time of application and type of electrode.

A

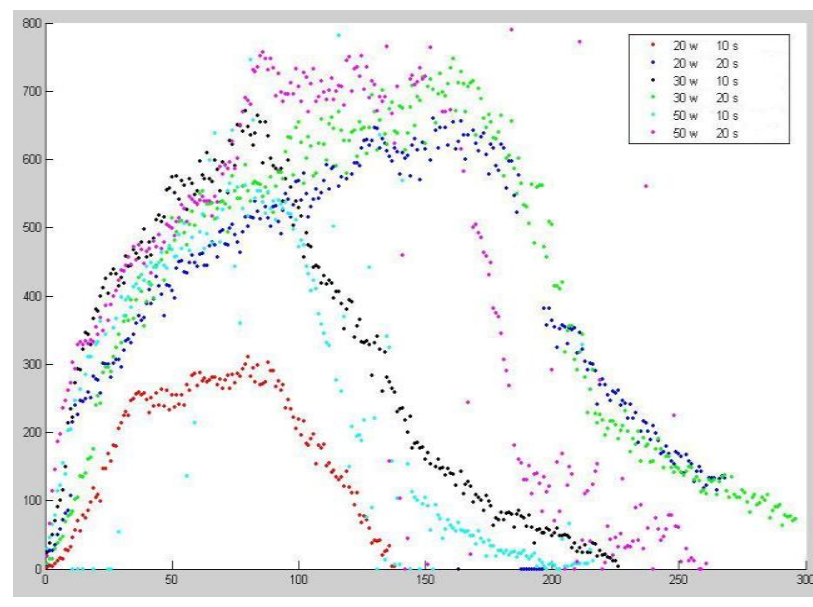

B

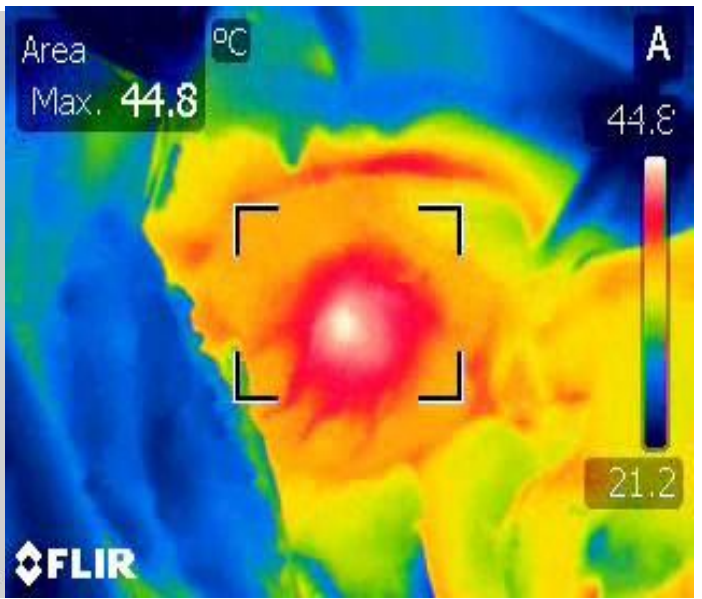


Pic.1. Total approximation curve from various $R F$ energy powers used in site of pulmonary veins $(A)$ and thermal view of RFA in right ventricle (B).

Peculiarities of RFA in site of pulmonary veins were similar comparing to RFA in wall of atrium, however damage area was $20-30 \%$ greater and site was as irregular ellipse pointed toward pulmonary veins flow to atrium. Temperature restoring in tissues also was similar, partial destruction zone depended on power of energy, time of application and type of electrode (bigger tip of electrode follows bigger site of damage). Approximation of various RF energy powers used in site of pulmonary veins (Pic.1A) worthy remark, that 30 and $50 \mathrm{~W} 20 \mathrm{~s}$ applications outcome is similar in total damage site, but partial damage site, as we saw in thermo grams - differs. Therefore, using RFA applications of $30 \mathrm{~W}$ and $20 \mathrm{~s}$ would be safe and optimal for damage site size. Applications that are more powerful can be not safe and cause strictures or stenosis of pulmonary veins, which in turn can cause embolisations and it was clearly observed in our experimental study.

Spread of RF energy in ventricle wall had specific peculiarities. We observed well-marked temperature dispersion and anisotropy belong wall of ventricle. Partial destruction zone went through myocardium and made $10-15 \mathrm{~mm}$ in width forming irregular oval with interstitial lines (Pic. 1B).

\section{Conclusions:}

Partial and atypical myocardial damage areas are significantly greater than was thought earlier. Interstitial damage phenomenon shows wide and inhomogeneous myocardial destruction including intracardial nervous structures and it could be useful in explaining the origin of most post-ablation complications. Thermography allows us to see the margins and features of destructive impact, optimize RFA parameters, due to avoiding and minimizing adverse effects and retain the structures that could influence traumatising outcomes. 